\title{
Collapse of the vacuum in hexagonal graphene quantum dots: a comparative study between the tight-binding and the mean-field Hubbard models
}

\author{
Mustafa Polat, ${ }^{1}$ Hâldun Sevinçli, ${ }^{2}$ and A. D. Güçlü ${ }^{1}$ \\ ${ }^{1}$ Izmir Institute of Technology, Department of Physics, 35430 Urla, Izmir, Turkey* \\ ${ }^{2}$ Izmir Institute of Technology, Department of Materials Science and Engineering, 35430 Urla, Izmir, Turkey
}

(Dated: May 22, 2020)

\begin{abstract}
In this paper, we perform a systematic study on the electronic, magnetic, and transport properties of the hexagonal graphene quantum dots (GQDs) with armchair edges in the presence of a charged impurity using two different configurations: (1) a central Coulomb potential and (2) a positively charged carbon vacancy. The tight binding (TB) and the half-filled extended Hubbard models are numerically solved and compared with each other in order to reveal the effect of electron interactions and system sizes. Numerical results point out that off-site Coulomb repulsion leads to an increase in the critical coupling constant to $\beta_{\mathrm{c}}=0.6$ for a central Coulomb potential. This critical value of the $\beta$ is found to be independent of the GQD size, reflecting its universality even in the presence of electron-electron interactions. In addition, a sudden downshift in the transmission peaks shows a clear signature of the transition from subcritical $\beta<\beta_{\mathrm{c}}$ to supercritical $\beta>\beta_{\mathrm{c}}$ regime. On the other hand, for a positively charged vacancy, collapse of the lowest bound state occurs at $\beta_{\mathrm{c}}=0.7$ for the interacting case. Interestingly, the local magnetic moment, induced by a bare carbon vacancy, is totally quenched when the vacancy is subcritically charged, whereas the valley splittings in electron and hole channels continue to exist in both regimes.
\end{abstract}

\section{INTRODUCTION}

The exact solution of 3D Dirac equation in an external Coulomb field, produced by a point nucleus, is only consistent up to a critical threshold $\mathrm{Z}_{\mathrm{c}}=\alpha^{-1} \sim$ 137, where $\alpha=e^{2} / \hbar c$ is the Sommerfeld fine-structure constant [1]. For larger values of the nuclear charge $\mathrm{Z}$, the energy eigenvalues become purely imaginary, the wave function is non-normalizable, and its real part exhibits oscillatory behavior 2. Actually, the singularity of the point nucleus at the center leads to a non-self-adjoint Hamiltonian that could not be properly solved unless a finite-size for the nucleus is introduced [3]. This regularization results in a larger critical threshold of $\mathrm{Z}_{c} \sim 172$ above which the wave function becomes a narrow resonance with a finite lifetime in compliance with Fano's formalism [4. In particular, the lowest bound state $1 \mathrm{~S}_{1 / 2}$ with the total angular momentum quantum number $j=1 / 2$ dives into the negative continuum for a coupling constant $\beta=\mathrm{Z} \alpha$ if it exceeds 1 , but the direct evidence of such a particular behavior has so far remained elusive in high energy heavy-ion collision experiments [5, 6]. However, the situation is slightly different in $2+1$ dimensions for which the critical coupling constant $\beta_{\mathrm{c}}$ becomes $1 / 2$ [7]. In this sense, bulk graphene with a larger fine structure constant $\alpha_{g}=2.2 / \kappa$, where $\kappa$ is the dielectric constant, could provide an ideal platform in theory [8]. Unlike $\mathrm{QED}, \mathrm{Z}_{c}$ is expected to be on the order of the unity [9, 10, which carries the signs of experimental transition to the supercritical regime in a table-top experiment. Indeed, the formation of an infinite family of quasi-bound states in the presence of

\footnotetext{
* mustafapolat@iyte.edu.tr
}

the clusters of charged calcium $(\mathrm{Ca})$ dimers on graphene have been successfully monitored via the local density of states (LDOS) in an experimental study [11.

Later, Mao et al. 12 demonstrated that a positive charge can be deposited into a single carbon vacancy by applying voltage pulses of $2-3 \mathrm{~V}$ for $>10$ seconds with the help of a scanning tunneling microscope (STM) tip. A charged vacancy in graphene is in analogy with the piling up positively charged ions and similarly leads to the sudden appearance of a sequence of quasi-bound states [12. Besides, it is well-known that the removal of a single carbon atom lifts the spin and valley degrees of freedom [8], hence the local magnetic moment is induced [13]. Since only a prominent resonant peak is observed in previous studies [14, 15], the spin splitting has recently attracted attention in experiments [16, 17. In addition, for a while there has been significant progress in measurement of the valley splittings around a carbon vacancy [18] thanks to discrete energy levels and an unconventional method of preparation of graphene quantum dots (GQDs) [19, 20. The question arises as to what sort of changes in physical properties happen after a bare vacancy is positively charged with the subcritical and supercritical Coulomb potentials.

Of all the GQDs that have been reported so far [2134, the hexagonal GQDs with armchair edges deserve attention due to the specific band gap feature. Free of localized edge states, the band gap is proportional to the inverse square root of number of atoms $\left(\mathrm{E}_{\text {gap }} \propto\right.$ $k_{\text {min }} \approx 2 \pi / \Delta x \propto 1 / \sqrt{\mathrm{N}}$ ) [31]. It corresponds to linear photon dispersion relation for confined Dirac fermions [35. In addition, the hexagonal shaped GQDs have wellknown properties, among which, (1) sublattice symmetry results in spin symmetry; (2) two doubly degenerate levels in the vicinity of Fermi level account for the valley symmetry [34]. These internal properties indicate 
that the pristine hexagonal GQDs with armchair edges carry all the symmetries of graphene. Therefore, it becomes possible to follow the evolution of the spin and valley splittings as a function of the coupling constant $\beta$ when a point vacancy is deliberately created and gradually charged. In this work, we construct a set of Dirac vacuums with the help of the hexagonal GQDs that differ in size. The tight-binding (TB) and the extended mean-field Hubbard (MFH) models are separately solved for the central Coulomb potential and a charged vacancy. The central impurity in a GQD was previously investigated using effective mass approach with appropriate boundary conditions [36, but Hubbard descriptions including electron-electron interactions and spin effects are still missing.

The rest of this paper is organized as follows. In Sec. II Hamiltonian of the extended MFH model, the Coulomb potential, and the non-equilibrium Green function formalism are introduced. The finite-size effect, the effect of electron-electron interactions, and the transmission coefficient of the lowest bound states for the central potential are discussed in greater detail in Sec. III. Both electronic and magnetic properties, as well as the transmission coefficients are studied in the presence of a charged vacancy in Sec. IV] Finally, Sec. V consists of our conclusions.

\section{COMPUTATIONAL METHODS}

We solve the extended mean-field theory of the Hubbard model starting from a single-band tight-binding approximation for the $\pi_{z}$ orbitals. The single-valley Dirac description of the $\pi_{z}$ electron dynamics are described by the following effective Hamiltonian

$$
\begin{aligned}
\mathrm{H}_{M F H} & =t \sum_{<i j>\sigma}\left(c_{i \sigma}^{\dagger} c_{j \sigma}+\text { H.c. }\right) \\
& +\mathrm{U} \sum_{i \sigma}\left(<n_{i \bar{\sigma}}>-\frac{1}{2}\right) n_{i \sigma} \\
& +\sum_{i j} \mathrm{~V}_{i j}\left(<n_{j}>-1\right) n_{i} \\
& -\hbar v_{\mathrm{F}} \beta \sum_{i \sigma} \frac{c_{i \sigma}^{\dagger} c_{i \sigma}}{r_{i}}
\end{aligned}
$$

The operator $\mathrm{c}_{i \sigma}^{\dagger}\left(\mathrm{c}_{j \sigma}\right)$ given in the first term describes the creation (annihilation) of an electron with spin $\sigma$ at the lattice sites. The nearest neighbor hopping term $t$ $=-2.8 \mathrm{eV}$ is used, and which preserves the electron-hole symmetry in the absence of the Coulomb potential 37. The second term is the on-site interaction term in which only two electrons with opposite spin can occupy the same lattice site by paying an extra correlation energy of $\mathrm{U}$. It is taken to be $16.52 / \kappa \mathrm{eV}$, where the dielectric constant $\kappa$ equals to 6 corresponding to the interband polarization [34, 38. In Eq. 11, the terms $\left\langle\mathrm{n}_{i \sigma}\right\rangle$ and $\mathrm{n}_{i \sigma}$ are associated with the spin dependent expectation value of electron densities and the number operator for an electron with spin $\sigma$ at the site i, respectively. The off-site Coulomb repulsion on top of the on-site repulsion is added to Hamiltonian by means of $\mathrm{V}_{i j}$ term which is set to be as $8.64 / \kappa \mathrm{eV}, 5.33 / \kappa \mathrm{eV}$, and $1 / \kappa \mathrm{d}_{i j} \mathrm{eV}$ for the first neighbors, second neighbors, and the atomic sites at relatively large distances, respectively 34, 39. The last term stands for the Coulomb potential in which $\mathrm{r}_{i}$ is the distance between the lattice site $i$ and the center of the Coulomb potential [40, and $v_{\mathrm{F}}$ is the Fermi velocity. The coupling constant $\beta$ is assumed to be attractive ( $>$ 0 ) without loss of generality in this study.

As a measurable feature of the critical states, LDOS is obtained by the formula

$$
\mathrm{N}(\mathrm{E}, \mathrm{r})=\sum_{n}\left|\Psi_{n}(r)\right|^{2} \delta\left(\mathrm{E}-\mathrm{E}_{n}\right) .
$$

LDOS is projected onto the lattice sites to demonstrate spatial distribution of the collapse states at different coupling constants. In the presence of a single charged vacancy, starting from the self-consistent expectation values of electron densities, we compute the spin density per lattice site as follows

$$
<s_{i}^{z}>=m_{i}=\left(<n_{i \uparrow}>-<n_{i \downarrow}>\right) / 2,
$$

where $\left\langle\mathrm{n}_{i \sigma}>\right.$ 's are calculated by summing up all states lying below Fermi level. Starting from Eq. 3, the staggered magnetization as an order parameter of the antiferromagnetism is numerically calculated from

$$
\mu_{s}^{z}=\sum_{i}(-1)^{i}<s_{i}^{z}>
$$

where $(-1)^{i}$ indicates that the contributions are summed up from the opposite sublattices with opposite signs. $\mu_{s}^{z}$ is proportional to the antiferromagnetic phase [4].

To calculate the transmission coefficients, we utilize the non-equilibrium Green function (NEGF) formalism. The transmission coefficients are obtained from

$$
\mathrm{T}(\mathrm{E})=\operatorname{Tr}\left(\Gamma^{L}(\mathrm{E}) \mathrm{G}(\mathrm{E}) \Gamma^{R}(\mathrm{E}) \mathrm{G}^{\mathrm{T}}(\mathrm{E})\right),
$$

where

$$
\mathrm{G}(\mathrm{E})=\left(\left(\mathrm{E}+i 0^{+}\right) \mathrm{I}_{\mathrm{N} \times \mathrm{N}}-\mathrm{H}_{c}-\Sigma_{L}-\Sigma_{R}\right)^{-1}
$$

is the Green function in which $0^{+}$is $10^{-6} \times t, \mathrm{H}_{c}$ represents the central Hamiltonian of the analyzed structures, and $\Sigma_{L}\left(\Sigma_{R}\right)$ is the self energy matrix of the left (right) probe, where generic electrodes are used in order to avoid structural features arising from the electrodes in the resulting transmission spectra. For that purpose, a one-dimensional wide bandwidth tightbinding chain is assumed. Self energies matrices $\left(\Sigma_{N \times N}\right)$ for the right and left leads are obtained from the 
analytical solution of surface Green function [42]. The probes are placed at the ends of the GQDs and the hopping term is taken as t/4. In Eq. 5. $\Gamma^{L, R}$ 's are the corresponding broadening matrices, and the hopping parameter of $t$ in the reservoirs is used 42. The transmission coefficients around the resonance energies of the defect-induced and atomic collapse states are numerically calculated for different values of the $\beta$.

\section{CENTRAL COULOMB POTENTIAL}

\section{A. Size quantization and electron-electron interactions}

To reveal the effect of the size quantization, we systematically study a series of the pristine hexagonal GQDs consisting of up to 10,806 atoms $(R=10.4 \mathrm{~nm})$. After this limit, physical properties approach to those of the corresponding bulk material 43. A Coulomb potential is placed at the center of each hexagonal GQDs; see the inset of Fig. 11(a). To discuss the size effect within the MFH model, energy eigenvalues of the lowest bound states of all samples as a function of the coupling strength $\beta$ and zoomed portion around the critical coupling constant $\beta_{c}$ are shown in Fig. 1(a) and Fig. 1(b), respectively. In Fig. 1(a) and (b), each of the lowest angular momentum channels is doubly degenerate due to the valley symmetry [28, 31]. In short, the spin and valley degeneracies are preserved as a function of the $\beta$. As a result, the MFH results do not make any discrimination between the spin components due to the spin symmetry. From now on, TB results are given by the black lines, while results of the spin-up and the spindown can be followed by the red and blue lines in each of the remaining graphs, respectively. Different kinds of symbols in Fig. 11(a) show the size of the hexagonal GQDs, and we also use these symbols in the remaining part of the paper.

Each of the lowest bound states dives into the negative energies at the same value of the coupling strength that is 0.6. It can be accepted as a critical point at this stage, and we will discuss this point in more detail below. It is clear that effect of the size is negligible due to the special characteristic of their band gaps. The collapse states are pinned at the Dirac point (DP) as clearly shown in the experiments [11, 12. In this sense, our results indicate that the zero energy plays the same role with the DP in bulk graphene. In contrast, Fermi level follows the highest filled level due to a constant number of electron-like Dirac fermions. Our results pave the way for the examination of reconstruction of the Dirac vacuum within quite small sample sizes by a low computational cost.

On the other hand, the electron-electron interactions in half-filled MFH model are set by the on-site $\mathrm{U}$ and off-site $\mathrm{V}$ terms as given in Eq. 1. Energy eigenvalues of TB model are compared with those of the MFH model
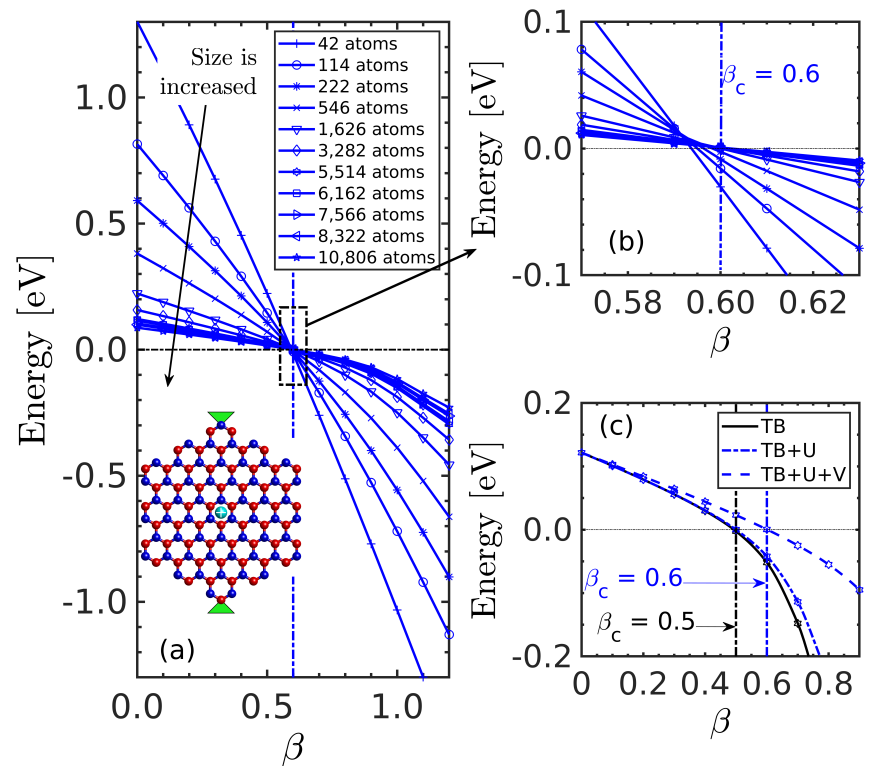

FIG. 1. Energy values of the lowest bound states as a function of the coupling constant $\beta$. (a) The critical coupling constant $\beta_{c}$ is 0.6 within the the MFH model for all samples that differ in size. The inset contains a sketch of the problem for the hexagonal GQD that consists of 114 atoms. Here the sublattices $\mathrm{A}$ and $\mathrm{B}$ are red and blue filled circles, and a positively charged impurity is at the center. Green triangles show how the leads are connected to samples throughout our study to determine the transmission coefficients. (b) shows a zoomed view of the energy eigenvalues. (c) contains a comparison between the TB and the MFH models for a GQD consisting of 5,514 carbon atoms.

by setting the off-site term $\mathrm{V}$ to zero. As it is clear from Fig. 1(c), the on-site term $U$ gives no contribution to the renormalization of the $\beta_{c}$. In contrast, the off-site term $\mathrm{V}$ decreases overscreening tendency [44, 45] of the TB approximation by smearing out the induced charge density [46, and which turns out to be a $20 \%$ increase in the $\beta_{c}$. It would be interesting to study screening properties in the GQDs as extensively examined in bulk graphene [46 52, but we directly give a critical bare valance charge $Z_{c}$

$$
\mathrm{Z}_{c}\left(\frac{2.2}{\kappa}\right)=\beta_{c} \quad \rightarrow \quad \mathrm{Z}_{c} \approx 1.64
$$

where the dielectric constant $\kappa=6$, and the critical coupling constant $\beta_{c}$ equals to 0.6. It indicates that impurities with the critical valence charge $\mathrm{Z}_{\mathrm{c}} \approx 1.64 \mathrm{can}$ be used to create an artificial supercritical nuclei for all GQD sizes. Our result is also consistent with the previous one in which $\mathrm{Z}_{\mathrm{c}}$ is calculated to be larger than unit charge [10. The tight-binding result for one particular hexagonal GQD consisting of 5,514 atoms shows (Fig. $1(\mathrm{c}))$ that the lowest bound state enters the supercritical regime at $\beta_{\mathrm{c}}=0.5$, same as what is expected for bulk graphene. In compliance with our results, the critical 
wave functions of the circular GQDs merge into negative energies at the value of $\beta_{\mathrm{c}}=0.5$ within the effective mass approximation with an infinite mass boundary condition 36].

The band gap in the GQDs is only due to size restriction of massless Dirac fermions, and we give an interaction-induced renormalization of the $\beta_{\mathrm{c}}$. This gap should not be confused with that of a gapped graphene monolayer [53], modelled by adding a mass term in bulk

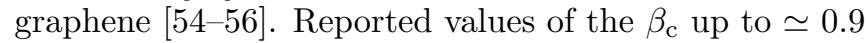
[57, 58] are calculated for the non-interacting massive Dirac fermions, where the critical point is defined as the crossing of the collapse state with the lower continuum [57, instead of DP in our calculations. In addition, Fermi level moves automatically down due to the absence of charge compensation, similar to our case.

\section{B. Transmission coefficients}

The transmission coefficients $\mathrm{T}$ of the lowest bound states as a function of the energy $\mathrm{E}$ are shown in Fig. 2 (a), (b), and (c) for the hexagonal GQDs consisting of $546,1,626$, and 10,806 atoms, respectively.
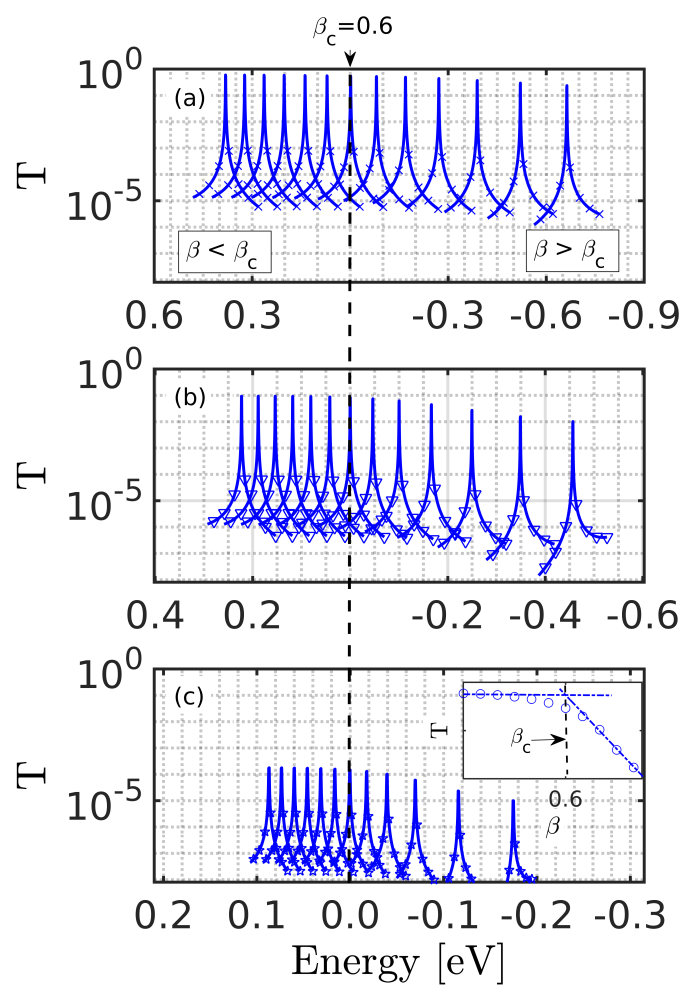

FIG. 2. The transmission coefficients in (a), (b), and (c) for the number of 546, 1,626, and 10,806 atoms, respectively. The behaviour of transmission coefficients obviously corresponds to two different regime. Inset in (c): the critical coupling constant $\beta_{\mathrm{c}}$ is at the point of intersection of two lines on a linear scale.
In all figures, from left to right, each of the transmission peaks is calculated for the consecutive values of the $\beta$ with a step size of 0.1 , starting from the $\beta=0$. When the subfigures are compared with each other in the absence of the Coulomb potential, i.e., $\beta$ $=0$, it is clear that the transmission coefficients of the lowest bound spin-down states decrease inversely with the size of the GQDs and reaches its minimum for the GQD that contains 10,806 atoms. It can be noted that the maximum transmission is observed for the all GQDs consisting of up to 222 atoms. For the subcritical range $0<\beta<\beta_{\mathrm{c}}$, the transmission coefficients do not make significant changes. In other words, the transmission coefficients of the lowest bound states remain almost the same in the subcritical regime due to the absence of the backscattering in the presence of the central Coulomb impurity [59].

When the coupling constant exceeds the critical value of $\beta_{\mathrm{c}}=0.6$, those coefficients drop immediately because of the collapse of the wave functions. The peak values of the transmission coefficients are plotted as a function of the coupling strength $\beta$ in the inset of Fig. 22(c) for the GQD consisting of 10,806 atoms. Two different regime are represented with the lines, and the point of intersection clearly exhibits the $\beta_{\mathrm{c}}$.

\section{CHARGED VACANCY}

\section{A. Spin and valley splittings}

The breaking of the four-fold symmetry in nanographene and related structures is a vital importance in understanding the electronic as well as magnetic properties [60. In this sense, we analyze the sublattice-induced symmetry breaking staring from the pristine hexagonal GQDs. DOS obtained for the clean hexagonal GQD consisting of 5,514 atoms using the TB model shows that the highest (lowest) occupied (unoccupied) state in the valence (conduction) band is doubly degenerate (Fig. 3(a)). It can be noted that all sizes have the same valley symmetry [34], and the valley degeneracy is observed in both the $\mathrm{TB}$ and the $\mathrm{MFH}$ models in the same way.

When a single carbon defect is intentionally created by removing of the $\pi_{z}$ orbital of the sublattice $\mathrm{A}$ from the central benzene, the broken symmetry of the valley states shows itself as the valley splittings with equal magnitude in electron and hole channels within the TB method, see the vertical arrows in Fig. 3(b). At Fermi level, we have a pronounced vacancy peak due to intervalley scattering caused by a bare carbon vacancy.

This vacancy state splits into up and down vacancy states with equal spin probability and the occupation of $\left\langle\mathrm{n}_{\downarrow}\right\rangle=1$ and $\left\langle\mathrm{n}_{\uparrow}\right\rangle=0$ as shown in Fig. 3.(c) when the interactions are turned on. These vacancy peaks are located symmetrically with respect to Fermi level, and the spin splitting $\Delta_{\text {spin }}$ between them is found 


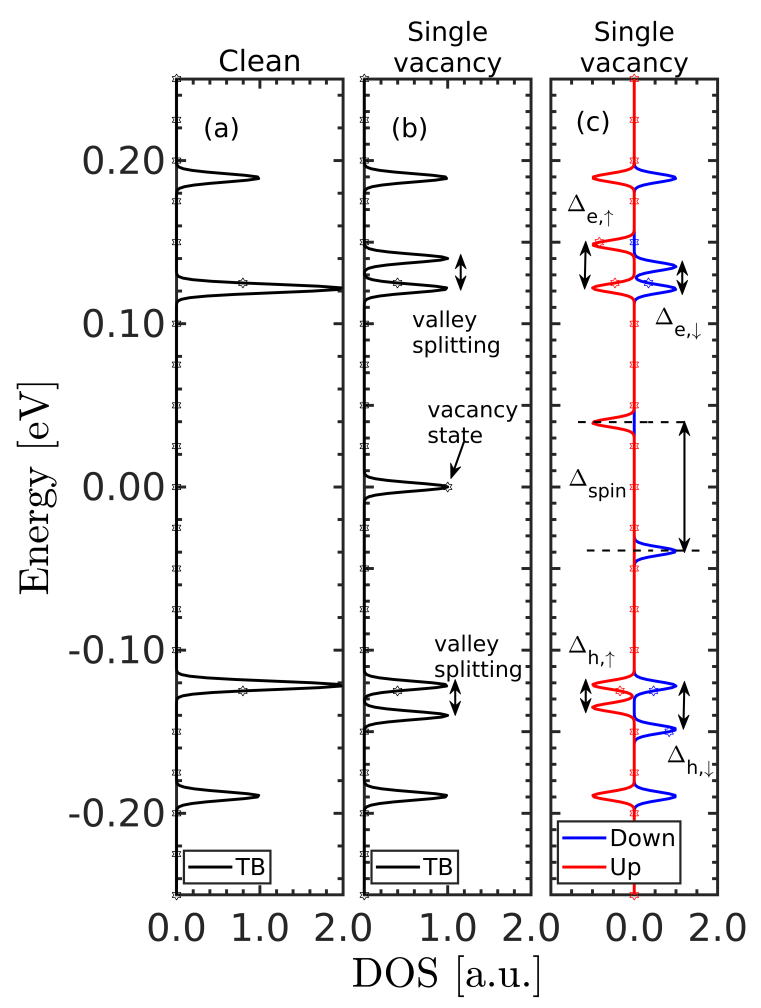

FIG. 3. (a) total densities of states are shown for a pristine hexagonal GQD consisting of 5,514 atoms, (b) total TB DOS belongs to the same GQD that contains a bare vacancy placed near the center, and (c) contains the spin and valley splittings for both spin components.

to be $78 \mathrm{meV}$ for this particular GQD. This splitting, also known as the spin polarization, is proportional to the on-site Coulomb repulsion U [17. When it comes to the valley splittings, the picture becomes much more complicated. Note that the total DOS distribution of the spin-up contains two unequivalent valley splittings. In the electron channel, we have the valley splitting $\Delta_{e, \uparrow}$ of $26 \mathrm{meV}$. In the hole channel, the valley splitting $\Delta_{h, \uparrow}$ is found to be $13 \mathrm{meV}$. Similarly, the total DOS distribution of the spin-down has two unequivalent valley splittings in both channels. Interestingly, there is an additional symmetry related to the valley splittings dictated by the electron-hole symmetry. In the presence of a bare vacancy on the A sublattice, that is given by

$$
\Delta_{\mathrm{e}, \downarrow}=\Delta_{\mathrm{h}, \uparrow}, \quad \Delta_{\mathrm{h}, \downarrow}=\Delta_{\mathrm{e}, \uparrow}
$$

As yet there is no discussion on the effect of the size on the splittings. To analyze the size dependence, the spin and valley splittings are plotted as a function of the size of the hexagonal GQDs in Fig. 4(a). It is clear that the valley splittings dominate the spin splitting at small sizes. On the contrary, for larger sizes, the valley splittings are quite small as compared to the spin splitting in the presence of a single bare vacancy. Moreover, the
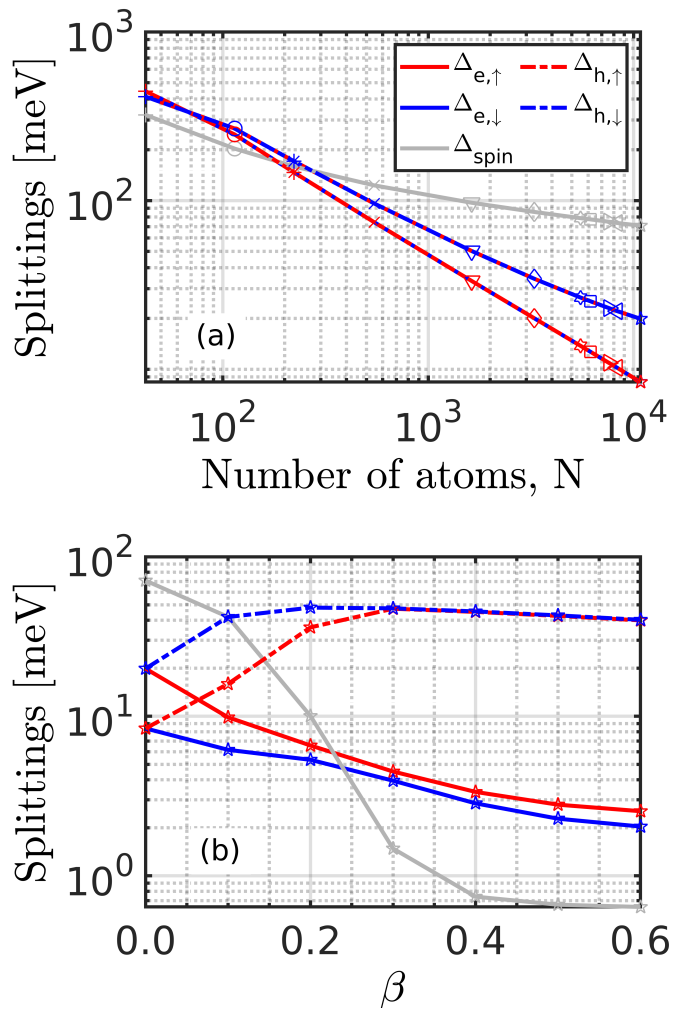

FIG. 4. (a) clearly shows the spin and valley splitings as a function the size of the hexagonal GQDs. Additional symmetry in Eq. 8 can be followed by the overlapped lines. (b) the spin splitting disappears as a function of $\beta$, while the valley splittings do not completely vanish.

additional symmetry between the valley splittings, given in Eq. 8, is conserved as a function of the size.

When the vacancy is positively charged with the Coulomb potential, the spin splitting decreases as a function the coupling strength $\beta$ as shown in Fig. 4(b). The quenching of the spin splitting occurs at the coupling constant of $\beta=0.4$ that lies in the subcritical regime. It mimics that the local magnetic moment can be tuned with the help of a charged vacancy.

The situation is totaly different in the valley splittings depending on the occupation of the states. While the valley splittings of $\Delta_{h, \uparrow}$ and $\Delta_{h, \downarrow}$ increase as a function of the coupling strength, both $\Delta_{e, \uparrow}$ and $\Delta_{e, \downarrow}$ show a decrement. However, all valley splittings continue to exist. As it is clear, the spin splitting has a different behaviour from that of the valley splittings for a charged vacancy, and which could prevent the valley states mixing with the spin states.

\section{B. State characteristics}

The TB energy spectrum of a GQD consisting of 5513 atoms is plotted in Fig. 5(a) as a function of the $\beta$. The vacancy state, labelled as (c) in Fig. 5(a), is pinned at 
the energy origin and dives immediately into negative energies when the carbon vacancy is charged. From top to bottom, the spatial distributions of the $\pi_{z}$-derived state are shown in Fig. 5(c) for the following values of the $\beta=0,0.1,0.2$, and 0.3 , respectively. When we zoomed into the bare defect (at the top of Fig. 5(c)), the triangular interference pattern due to intervalley scattering can be seen as a characteristic spatial shape 14. As the $\beta$ is increased, the intervalley scattering is gradually surpassed by the intra-valley scattering, and finally the uniform distribution of the vacancy state takes place at the $\beta=0.2$ and 0.3 . It means that highly localized defect state returns to its original bound state characteristic; however, these scaled figures render the uniform spatial distribution invisible. This particular behaviour will be strengthened below by means of the transmission coefficients.

It is also shown the spatial extension of the state labeling as (d) in Fig. 5(a). From top to bottom, Fig. 5(d) exhibits the spatial extension of the critical state around the vacancy for the $\beta=0,0.3,0.6,0.8$, and 1.0 , respectively. Uniform spatial extension of the critical state exists for the $\beta=0$ as shown at the top of Fig. 5(d).
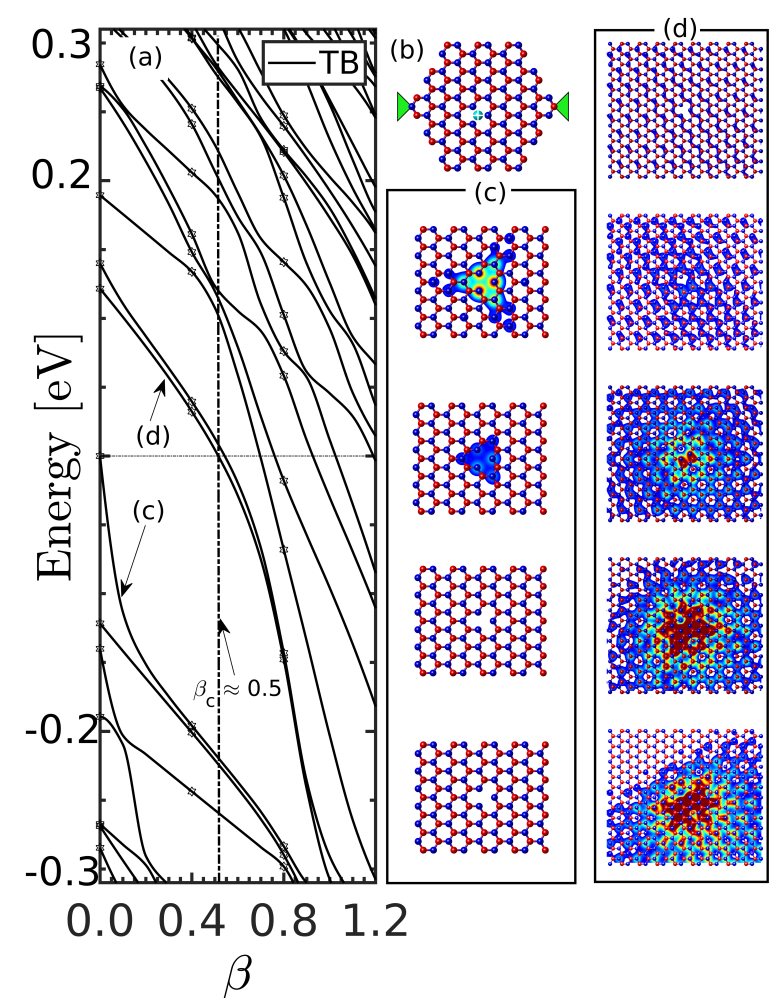

FIG. 5. The energy spectrum of TB model as a function of the $\beta$ is shown in (a). The positions of the leads and the bare carbon vacancy are sketched in (b). Scaled electronic densities per lattice of the vacancy state, i.e., LDOS, for the $\beta=0,0.1$, 0.2 , and 0.3 can be seen in (c), from top to bottom. The quasilocalization of the lowest bound state is demonstrated in (d) for the $\beta=0,0.3,0.6,0.8$, and 1.0 , from top to bottom.
On exceeding the critical value, the $\beta \gtrsim 0.5$, the critical state dives into negative energy spectrum, so that the appearance of the quasi-localized state occurs around the charged vacancy. It is actually defined as the counterpart of the 1S atomic collapse state in Ref. [12.

When the electron interactions are turned on, we have a different picture. The energy spectrum of the spin-up is superimposed to that of the spin-down in Fig. 6(a) as a function of the $\beta$. If Fig. 6(a) is analyzed, the spin symmetry does not exist up to the $\beta=0.4$. In other words, an exact overlap of the energy spectrums occurs at the $\beta=0.4$ meaning that the spin symmetry is regained for the larger coupling strength values as previously discussed.

There is a defect state in the spin-up spectrum that is labelled as (b) in Fig. 6(a). Its spatial distribution is displayed in Fig. 6(b) for the $\beta=0,0.2$, and 0.4 starting from the top. The defect state in the spin-up spectrum merges into negative energies when the $\beta$ exceeds 0.1 . The ideal triangular interference pattern characteristic starts to decay, indicating a uniformly distribution on the lattice sites. On the other hand, the defect state in the spin-down spectrum loses its triangular shape from

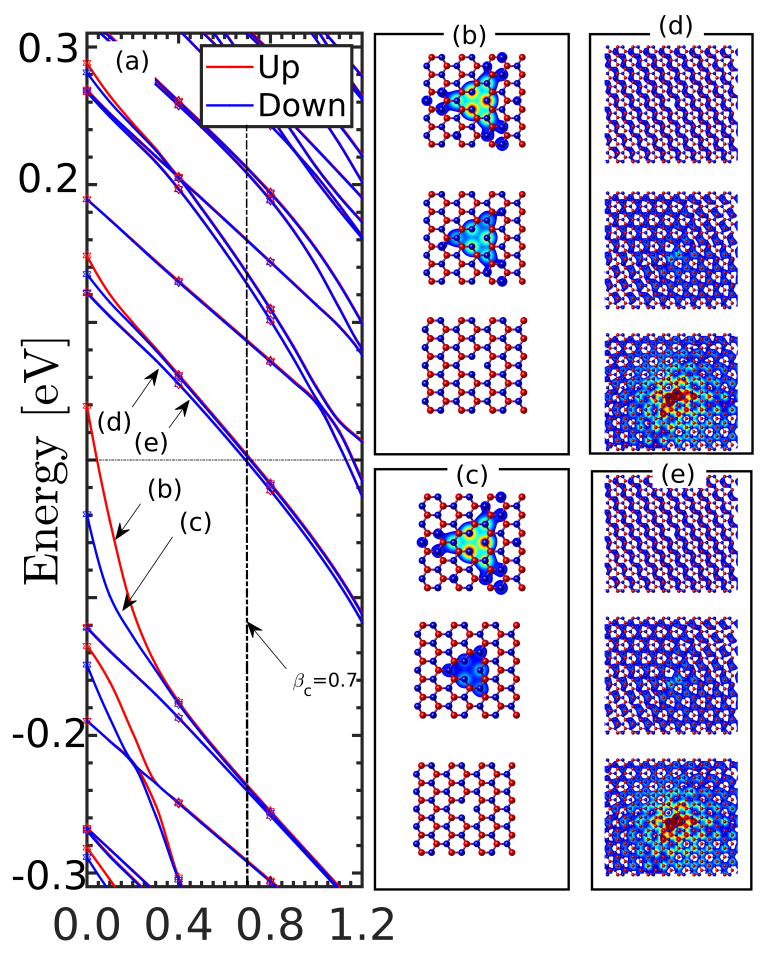

$\beta$

FIG. 6. The energy spectrums of the spin-up and spin-down are shown in (a). The $\beta_{c}$ equals to 0.7 for a Coulomb charged vacancy. Scaled electronic densities for the vacancy states can be seen in (b) and (c) for the $\beta=0,0.2$, and 0.4 , from top to bottom. In (d) and (e), the behaviour of the critical states for the $\beta$ values of $0,0.7$, and 1.2 can be seen starting from top. 
the moment the vacancy begins to charge, and similarly it has a uniform distribution at the $\beta=0.4$ as shown at the bottom of Fig. 6(c). At a value of the $\beta=0.7$, both spectrums have new diving levels; see in Fig. 6(a). Both of the critical states become quasi-localized in the supercritical regime as displayed in the right columns for spin-up (d) and spin-down (e) states for the $\beta=0,0.7$, and 1.2, from top to bottom, respectively. As compared to the non-interacting case, the critical coupling constant is renormalized to the $\beta_{c}=0.7$ in the presence of electronelectron interactions. The critical states in both energy spectrums collapse at the same $\beta_{c}$. The values of the $\beta_{c}$ are valid for all sizes of the hexagonal GQDs when a vacancy is charged with the Coulomb potential.

\section{Transmission coefficients and staggered magnetization}

Transmission coefficients of the critical states of TB, spin-up, and spin-down spectrums are calculated. First of all, in Fig. 7(a), (c), and (e), the transmission coefficients are approximately $2 \times 10^{-4}$ in the subcritical regime $\beta<\beta_{c}$. It can be inferred that there is no a direct effect of including electron-electron interactions on the transmission coefficients. Whenever a critical state dives into the negative energies, which happens at the $\beta_{c} \approx$ 0.5 for TB and $\beta_{c}=0.7$ for the MFH spectrums, the transmission coefficients immediately drop. Basically, the quasi-localized character of these states is responsible for a decrement observed in transmission coefficients.

The transmission coefficient of the vacancy state in TB spectrum is plotted in Fig. 7(b). It has too small transmission value at the $\beta=0$, whereas the transmission coefficient increases and stays nearly the same for the $\beta$ $>0.1$.

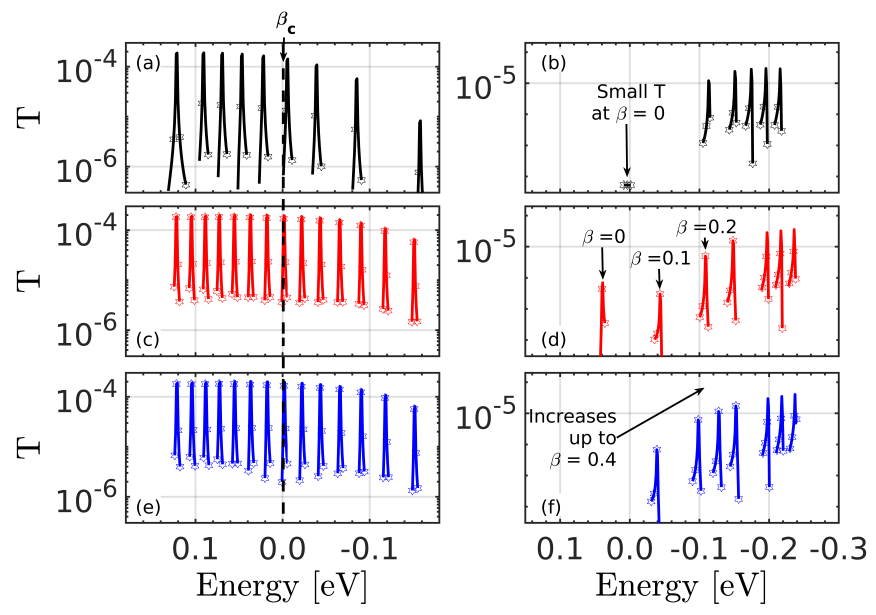

FIG. 7. Transmission coefficients of the critical states of TB in (a), spin-up in (c), and spin-down in (e) spectrums, while the vacancy states are given in $(b),(d)$, and (f).
This result actually points out that returning to the bound state characteristic leads to an increase in the transmission coefficient (see again Fig. 5(c)). The same physics is valid for all the vacancy states observed within the MFH models. As shown in Fig. 77(d), the transmission coefficient for the vacancy state in the spin-up spectrum reaches its maximum at the $\beta$ $=0.4$, although there is a small deviation at the $\beta=$ 0.1 . When it comes to the vacancy state in the spindown spectrum, the transmission coefficient (Fig. 7(f)) gradually increases up to the $\beta=0.4$ when we charge the defect. The reason for this is the recovering of the initial bound state characteristic.

As plotted in Fig. 8, a large amount of the staggered magnetization $\mu_{s}^{z}$ vanishes when the coupling constant $\beta$ equals to 0.4. This behaviour guarantees that the spin symmetry is regained for a Coulomb charged vacany. In this manner, the mechanisms of evolution, observed for the vacancy states in Fig. 6(b) and (c), seem to be the underlying reason.

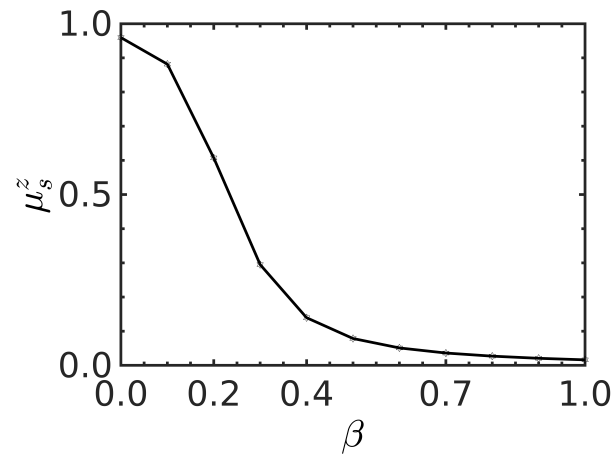

FIG. 8. The quenching of staggered magnetization $\mu_{s}^{z}$ is given as a function of the $\beta$. Up to the $\beta=0.4$, a large portion of the $\mu_{s}^{z}$ disappears.

\section{CONCLUSIONS}

In summary, the hexagonal GQDs with special characteristic band gap feature are studied by means of TB and MFH models. The critical coupling constant is found to be $\beta_{c}=0.5$ for the non-interacting cases when the Coulomb potential is placed at the center. However, the $\beta_{c}$ is renormalized to 0.6 for all sizes in the presence of off-site electron-electron interactions. It can be noted that the off-site repulsion term is responsible for this incasement due to the long-range repulsive tail. It is calculated that the central impurities with bare nuclear charge $\mathrm{Z}_{\mathrm{c}} \approx 1.64$ are at the edge of the supercritical threshold. Additionally, it is revealed that the transmission coefficients remain the same in the subcritical regime $\beta<\beta_{c}$ due to the absence of the backscattering. However, those values in the supercritical regime $\beta>\beta_{c}$ shows a strong dependence 
on the coupling strength.

It is revealed with the help of DOS that a bare vacancy gives rise to the simultaneous formation of the valley and spin splittings. The spin splitting is larger than the valley splittings for the larger sizes, whereas the valley splittings become dominant for the small sizes. As the coupling strentgh $\beta$ is increased, the spin splitting vanishes at $\beta=0.4$. The behaviour of valley splittings completely depends on the occupation of the valley states. In the hole (electron) channel, the valley splittings show an increment (decrement) for the larger coupling strength. However, the valley splittings never vanish. It signals that the mixing of the valley states with the spin states is not possible in the presence of a charged vacancy.

The formation of the quasi-localization around a charged vacancy is monitored with the help of LDOS. The critical state collapses when the coupling constant exceeds $\beta_{c} \approx 0.5$ for $\mathrm{TB}$ and $\beta_{c}=0.7$ for the $\mathrm{MFH}$ models for a charged vacancy. Furthermore, the transmission coefficient of the critical states decreases in the supercritical regime. On the contrary, those coefficients of the vacancy states increase in the subcritical regime, as the coupling strength is increased. The quenching of the spin splitting is also discussed with the help of the staggered magnetization which reinforces the findings related to regaining of the spin symmetry.

\section{ACKNOWLEDGMENTS}

We are grateful to A. M. Altıntaş and P. Hawrylak for stimulating discussions and to A. M. Altıntaş for helping with computational algorithms. This work was supported by The Scientific and Technological Research Council of Turkey (TUBITAK) under the 1001 Grant Project Number 116F152.
[1] Y. B. Zeldovich and V. S. Popov, Electronic structure of superheavy atoms, Sov. Phys. Uspekhi 14, 673 (1972).

[2] W. Greiner et al., Relativistic Quantum Mechanics, Volume 3 (Springer, 1990).

[3] I. Pomeranchuk and Y. Smorodinsky, On the energy levels of systems with Z > 137, J. Phys. Ussr 9, 97 (1945).

[4] J. Reinhardt and W. Greiner, Quantum electrodynamics of strong fields, Rep. Prog. Phys. 40, 219 (1977).

[5] J. Schweppe, A. Gruppe, K. Bethge, H. Bokemeyer, T. Cowan, H. Folger, J. Greenberg, H. Grein, S. Ito, R. Schule, et al., Observation of a peak structure in positron spectra from U+Cm collisions, Phys. Rev. Lett. 51, 2261 (1983).

[6] T. Cowan, H. Backe, M. Begemann, K. Bethge, H. Bokemeyer, H. Folger, J. Greenberg, H. Grein, A. Gruppe, Y. Kido, et al., Anomalous positron peaks from supercritical collision systems, Phys. Rev. Lett. 54, 1761 (1985).

[7] V. Khalilov and C.-L. Ho, Dirac electron in a coulomb field in $(2+1)$ dimensions, Mod. Phys. Lett. A 13, 615 (1998).

[8] A. C. Neto, F. Guinea, N. M. Peres, K. S. Novoselov, and A. K. Geim, The electronic properties of graphene, Rev. Mod. Phys. 81, 109 (2009).

[9] A. Shytov, M. Katsnelson, and L. Levitov, Atomic collapse and quasi-Rydberg states in graphene, Phys. Rev. Lett. 99, 246802 (2007).

[10] I. S. Terekhov, A. I. Milstein, V. N. Kotov, and O. P. Sushkov, Screening of coulomb impurities in graphene, Phys. Rev. Lett. 100, 076803 (2008).

[11] Y. Wang, D. Wong, A. V. Shytov, V. W. Brar, S. Choi, Q. Wu, H.-Z. Tsai, W. Regan, A. Zettl, R. K. Kawakami, et al., Observing atomic collapse resonances in artificial nuclei on graphene, Science 340, 734 (2013).

[12] J. Mao, Y. Jiang, D. Moldovan, G. Li, K. Watanabe, T. Taniguchi, M. R. Masir, F. M. Peeters, and E. Y. Andrei, Realization of a tunable artificial atom at a supercritically charged vacancy in graphene, Nat. Phys. 12,545 (2016).
[13] O. V. Yazyev and L. Helm, Defect-induced magnetism in graphene, Phys. Rev. B 75, 125408 (2007).

[14] M. M. Ugeda, I. Brihuega, F. Guinea, and J. M. Gómez-Rodríguez, Missing atom as a source of carbon magnetism, Phys. Rev. Lett. 104, 096804 (2010).

[15] M. Ugeda, D. Fernández-Torre, I. Brihuega, P. Pou, A. Martínez-Galera, R. Pérez, and J. Gómez-Rodríguez, Point defects on graphene on metals, Phys. Rev. Lett. 107, 116803 (2011).

[16] Y. Zhang, S.-Y. Li, H. Huang, W.-T. Li, J.-B. Qiao, W.X. Wang, L.-J. Yin, K.-K. Bai, W. Duan, and L. He, Scanning tunneling microscopy of the $\pi$ magnetism of a single carbon vacancy in graphene, Phys. Rev. Lett. 117, 166801 (2016).

[17] H. González-Herrero, J. M. Gómez-Rodríguez, P. Mallet, M. Moaied, J. J. Palacios, C. Salgado, M. M. Ugeda, J.Y. Veuillen, F. Yndurain, and I. Brihuega, Atomic-scale control of graphene magnetism by using hydrogen atoms, Science 352, 437 (2016).

[18] S.-Y. Li, Y.-N. Ren, Y.-W. Liu, M.-X. Chen, H. Jiang, and L. He, Nanoscale detection of valley-dependent spin splitting around atomic defects of graphene, 2D Mater. 6, 031005 (2019).

[19] N. M. Freitag, L. A. Chizhova, P. Nemes-Incze, C. R. Woods, R. V. Gorbachev, Y. Cao, A. K. Geim, K. S. Novoselov, J. Burgdörfer, F. Libisch, et al., Electrostatically confined monolayer graphene quantum dots with orbital and valley splittings, Nano Lett. 16, 5798 (2016).

[20] N. M. Freitag, T. Reisch, L. A. Chizhova, P. NemesIncze, C. Holl, C. R. Woods, R. V. Gorbachev, Y. Cao, A. K. Geim, K. S. Novoselov, et al., Large tunable valley splitting in edge-free graphene quantum dots on boron nitride, Nat. Nanotechnol. 13, 392 (2018).

[21] M. Ezawa, Metallic graphene nanodisks: Electronic and magnetic properties, Phys. Rev. B 76, 245415 (2007).

[22] J. Fernández-Rossier and J. J. Palacios, Magnetism in graphene nanoislands, Phys. Rev. Lett. 99, 177204 (2007). 
[23] W. L. Wang, S. Meng, and E. Kaxiras, Graphene nanoflakes with large spin, Nano Lett. 8, 241 (2008).

[24] J. Akola, H. Heiskanen, and M. Manninen, Edgedependent selection rules in magic triangular graphene flakes, Phys. Rev. B 77, 193410 (2008).

[25] S. Schnez, K. Ensslin, M. Sigrist, and T. Ihn, Analytic model of the energy spectrum of a graphene quantum dot in a perpendicular magnetic field, Phys. Rev. B 78, 195427 (2008).

[26] M. L. Mueller, X. Yan, J. A. McGuire, and L.-s. Li, Triplet states and electronic relaxation in photoexcited graphene quantum dots, Nano Lett. 10, 2679 (2010).

[27] M. Wimmer, A. Akhmerov, and F. Guinea, Robustness of edge states in graphene quantum dots, Phys. Rev. B 82, 045409 (2010).

[28] M. Zarenia, A. Chaves, G. Farias, and F. Peeters, Energy levels of triangular and hexagonal graphene quantum dots: a comparative study between the tight-binding and dirac equation approach, Phys. Rev. B 84, 245403 (2011).

[29] S. K. Hämäläinen, Z. Sun, M. P. Boneschanscher, A. Uppstu, M. Ijäs, A. Harju, D. Vanmaekelbergh, and P. Liljeroth, Quantum-confined electronic states in atomically well-defined graphene nanostructures, Phys. Rev. Lett. 107, 236803 (2011).

[30] M. Olle, G. Ceballos, D. Serrate, and P. Gambardella, Yield and shape selection of graphene nanoislands grown on $\mathrm{Ni}(111)$, Nano Lett. 12, 4431 (2012).

[31] W.-d. Sheng, M. Korkusinski, A. D. Güçlü, M. Zielinski, P. Potasz, E. S. Kadantsev, O. Voznyy, and P. Hawrylak, Electronic and optical properties of semiconductor and graphene quantum dots, Front. Phys. 7, 328 (2012).

[32] D. Subramaniam, F. Libisch, Y. Li, C. Pauly, V. Geringer, R. Reiter, T. Mashoff, M. Liebmann, J. Burgdörfer, C. Busse, et al., Wave-function mapping of graphene quantum dots with soft confinement, Phys. Rev. Lett. 108, 046801 (2012).

[33] K. Szalowski, Indirect coupling between localized magnetic moments in triangular graphene nanoflakes, Physica E 52, 46 (2013).

[34] A. D. Güçlü, P. Potasz, M. Korkusinski, and P. Hawrylak, Graphene Quantum Dots (Springer, 2014).

[35] A. Güçlü, P. Potasz, and P. Hawrylak, Excitonic absorption in gate-controlled graphene quantum dots, Phys. Rev. B 82, 155445 (2010).

[36] R. Van Pottelberge, M. Zarenia, P. Vasilopoulos, and F. Peeters, Graphene quantum dot with a coulomb impurity: Subcritical and supercritical regime, Phys. Rev. B 95, 245410 (2017).

[37] V. M. Pereira, J. Nilsson, and A. C. Neto, Coulomb impurity problem in graphene, Phys. Rev. Lett. 99, 166802 (2007).

[38] T. Ando, Screening effect and impurity scattering in monolayer graphene, J. Phys. Soc. Jpn. 75, 074716 (2006).

[39] P. Potasz, A. Güçlü, and P. Hawrylak, Spin and electronic correlations in gated graphene quantum rings, Phys. Rev. B 82, 075425 (2010).

[40] D. Moldovan and F. Peeters, Atomic collapse in graphene in Nanomaterials for Security (Springer, 2016).

[41] M. Grujić, M. Tadić, and F. Peeters, Antiferromagnetism in hexagonal graphene structures: Rings versus dots, Phys. Rev. B 87, 085434 (2013).

[42] W. Müller, R. Schiller, and W. Nolting, Understanding of surface states in a correlated electron system, Eur.
Phys. J. B 16, 705 (2000).

[43] M. Li, T. Chen, J. J. Gooding, and J. Liu, Review of carbon and graphene quantum dots for sensing, ACS sensors 4, 1732 (2019).

[44] V. N. Kotov, B. Uchoa, and A. C. Neto, Electron-electron interactions in the vacuum polarization of graphene, Phys. Rev. B 78, 035119 (2008).

[45] V. N. Kotov, B. Uchoa, V. M. Pereira, F. Guinea, and A. C. Neto, Electron-electron interactions in graphene: Current status and perspectives, Rev. Mod. Phys. 84, 1067 (2012).

[46] R. R. Biswas, S. Sachdev, and D. T. Son, Coulomb impurity in graphene, Phys. Rev. B 76, 205122 (2007).

[47] A. V. Shytov, M. I. Katsnelson, and L. S. Levitov, Vacuum polarization and screening of supercritical impurities in graphene, Phys. Rev. Lett. 99, 236801 (2007).

[48] O. Gamayun, E. Gorbar, and V. Gusynin, Magnetic field driven instability of a charged center in graphene, Phys. Rev. B 83, 235104 (2011).

[49] Y. Wang, V. W. Brar, A. V. Shytov, Q. Wu, W. Regan, H.-Z. Tsai, A. Zettl, L. S. Levitov, and M. F. Crommie, Mapping dirac quasiparticles near a single coulomb impurity on graphene, Nat. Phys. 8, 653 (2012).

[50] Y. Nishida, Vacuum polarization of graphene with a supercritical coulomb impurity: Low-energy universality and discrete scale invariance, Phys. Rev. B 90, 165414 (2014).

[51] A. Luican-Mayer, M. Kharitonov, G. Li, C.-P. Lu, I. Skachko, A.-M. B. Goncalves, K. Watanabe, T. Taniguchi, and E. Y. Andrei, Screening charged impurities and lifting the orbital degeneracy in graphene by populating Landau levels, Phys. Rev. Lett. 112, 036804 (2014).

[52] D. Wong, F. Corsetti, Y. Wang, V. W. Brar, H.-Z. Tsai, Q. Wu, R. K. Kawakami, A. Zettl, A. A. Mostofi, J. Lischner, et al., Spatially resolving density-dependent screening around a single charged atom in graphene, Phys. Rev. B 95, 205419 (2017).

[53] S. Y. Zhou, G.-H. Gweon, A. Fedorov, d. First, PN, W. De Heer, D.-H. Lee, F. Guinea, A. C. Neto, and A. Lanzara, Substrate-induced bandgap opening in epitaxial graphene, Nat. Mater. 6, 770 (2007).

[54] V. N. Kotov, V. M. Pereira, and B. Uchoa, Polarization charge distribution in gapped graphene: Perturbation theory and exact diagonalization analysis, Phys. Rev. B 78, 075433 (2008).

[55] B. Chakraborty, K. S. Gupta, and S. Sen, Effect of topological defects and coulomb charge on the low energy quantum dynamics of gapped graphene, J. Phys. A: Math. Theor. 46, 055303 (2013).

[56] V. M. Kuleshov, V. D. Mur, N. B. Narozhny, A. M. Fedotov, and Y. E. Lozovik, Coulomb problem for graphene with the gapped electron spectrum, JETP Lett. 101, 264 (2015).

[57] V. M. Pereira, V. N. Kotov, and A. C. Neto, Supercritical coulomb impurities in gapped graphene, Phys. Rev. B 78, 085101 (2008).

[58] W. Zhu, Z. Wang, Q. Shi, K. Szeto, J. Chen, and J. Hou, Electronic structure in gapped graphene with a coulomb potential, Phys. Rev. B 79, 155430 (2009).

[59] D. Novikov, Elastic scattering theory and transport in graphene, Phys. Rev. B 76, 245435 (2007).

[60] A. Altıntaş and A. D. Güçlü, Defect induced anderson 
localization and magnetization in graphene quantum dots, Solid State Commun. 281, 44 (2018). 